\title{
Sur l'entropie et le paysage : à propos de Robert Smithson
}

Jacques Leenhardt

\section{CpenEdition}

12 Journals

Édition électronique

URL : https://journals.openedition.org/imagesrevues/3491

DOI : 10.4000/imagesrevues.3491

ISSN : $1778-3801$

Éditeur :

Centre d'Histoire et Théorie des Arts, Groupe d'Anthropologie Historique de l'Occident Médiéval, Laboratoire d'Anthropologie Sociale, UMR 8210 Anthropologie et Histoire des Mondes Antiques

\section{Référence électronique}

Jacques Leenhardt, « Sur l'entropie et le paysage : à propos de Robert Smithson », Images Re-vues [En ligne], Hors-série 5 | 2016, mis en ligne le 01 janvier 2014, consulté le 04 mars 2022. URL : http:// journals.openedition.org/imagesrevues/3491; DOI : https://doi.org/10.4000/imagesrevues.3491

Ce document a été généré automatiquement le 4 mars 2022.

Images Re-vues est mise à disposition selon les termes de la Licence Creative Commons Attribution Pas d'Utilisation Commerciale 4.0 International. 


\title{
Sur l'entropie et le paysage : à propos de Robert Smithson
}

\author{
Jacques Leenhardt
}

1 La deuxième guerre mondiale a laissé au cœur de l'histoire européenne un trop vaste désert. Du Havre à Dresde et Stalingrad, ce ne sont que champs de ruines à la surface du sol et dans les esprits, la bombe de Hiroshima a fait renaître l'hypothèse de l'Apocalypse. Les activités artistiques occupent une place singulière $\mathrm{du}$ mouvement politique et intellectuel, littéraire et esthétique qui

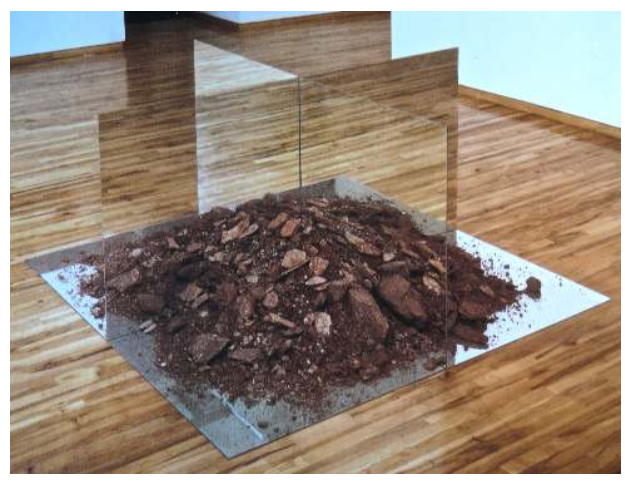
construisit, sinon des réponses, du moins des attitudes face à ces interrogations extrêmes auxquelles chacun eut sa manière de répondre.

Dans ces circonstances, les artistes ont souvent cherché dans l'effervescence des avantgardes du début du siècle l'inspiration et le vocabulaire susceptibles de les aider à réanimer une scène sinistrée dans le climat délétère des années de guerre. En adoptant un regard historien surplombant, tout semble se passer, dans les années qui suivent, comme si la grande parenthèse ouverte par la première guerre mondiale se refermait avec la fin de la seconde, et que l'esprit de 1913-1915 réapparaissait avec sa gouaille et son invention, son radicalisme et ses questionnements.

3 D'un côté fleurit une abondante production sur l'héritage du formalisme et de l'abstraction, dans laquelle Clement Greenberg verra l'accomplissement de la modernité picturale, de l'autre, des profondeurs de la catastrophe, remonte une interrogation radicale sur la tradition du tableau elle-même, où se précipite, dans une alchimie complexe, l'esprit dada et duchampien et les vapeurs de l'inconscient sur les braises duquel souffle toujours l'esprit surréaliste. 
4 C'est devant cette toile de fond trop rapidement brossée, comme un décor grossier où s'animent les figures de l'art des années quarante et cinquante, que je voudrais placer l'artiste qui retiendra mon attention : Robert Smithson.

5 Né peu avant la guerre Smithson (1938-1973) apparaît dans l'histoire de l'art à ce moment où les cendres de Hiroshima ont à peine fini de retomber sur une terre brûlée. Si ses yeux avaient eu le don d'ubiquité, il aurait vu sur le terrain européen : des Otages de Fautrier, des Texturologies de Dubuffet, des cires et des graisses de Beuys, toute une assomption du matériau, de l'infra pictural, une manière de degré zéro de la peinture. Pendant ce temps au contraire se développait, dans le climat plus serein des terres nord américaines qu'il fréquentait, une sorte d'apothéose de la peinture dans son jaillissement. Sans doute le tragique n'était pas loin, chez Pollock et De Kooning, mais finalement la peinture triomphait des angoisses et proposait une dramaturgie spectaculaire dont le pop art allait savoir tirer les profits. Les noces de la tradition picturale et de la modernité esthétisante, déjà proche des arts consommés du packaging, n'étaient pas loin d'être célébrées.

6 C'est à ce moment tournant que quelques artistes inventent un chemin de traverse. Ils veulent tourner le dos au cadre normé et contraignant du cube blanc de la galerie (et du musée) ${ }^{1}$ dont la tradition moderne avait fait l'écrin par excellence de l'art moderne. Sortir de la galerie, c'était aussi pour eux renoncer à la surface peinte et, dans une certaine mesure, échapper au cadre institutionnel qui avait de plus en plus réduit l'œuvre d'art à un objet esthétique de consommation.

7 Quelles que soient les interrogations que l'on peut avoir sur la signification du geste qui consistait à sortir de la galerie, cet éloignement eut lieu et ouvrit des champs de perception et d'interrogation inédits. Peut-être davantage encore que ses amis et complices de la première exposition de Earth Works (1969), Robert Smithson découvre, en sortant de l'espace normé de la galerie, le tumulte cataclysmique du «paysage ». Si pour Michael Heizer, Nancy Holt ou d'autres artistes qui peu à peu s'agrégèrent autour de la notion de Land Art, le paysage était une toile de fond sur laquelle l'artiste pouvait inscrire des formes, ce que Smithson découvre dans le "paysage ", c'est un univers en mouvement, travaillé de l'intérieur par un principe d'instabilité et d'usure permanente auquel la science avait donné un nom : entropie.

Le « paysage » devient pour Smithson ce qui cristallise les différents aspects de la crise esthétique de l'art. Penser ce qui s'offre au regard comme "paysage» lui permet d'emblée de conjurer la clôture qui affecte le monde de l'art, reclus en ses galeries et musées, contraint en ses institutions, épuisé par son endogamie et qui peine à se hausser aux grands vents de l'histoire. Infini et toujours renouvelé, appartenant aux catégories spatiales aussi bien que temporelles dans sa mue perpétuelle, le "paysage » fait exploser les cadres comme faisait l'exposition d'Yves Klein Le Vide à la Galerie Iris Clert en 1958.

Du même coup, la mise sous tutelle de plus en plus insidieuse de l'art par les lois du marché se trouve contournée. En refusant de produire des objets " vendables ", les land artistes participent au renouvellement de la définition même de l'artiste. Le résultat du travail artistique cessant d'être simplement un « objet d'art ", prêt pour la circulation mercantile, il constitue cependant ce que Duchamp avait appelé par provocation un " objet dard ", quelque chose, un objet peut-être, un geste ou une situation, qui peut atteindre son spectateur au cœur de sa sensibilité. Harald Szeemann tirera les 
conclusions de ce tournant en nommant sa fameuse exposition à la Kunsthalle de Berne: « Quand les attitudes deviennent forme. » (1969)

Une problématique nouvelle apparaît dans cette exposition. Celle-ci ne se réduit plus à la présentation d'objets, à l'exhibition de leur design, bref à la mise en public et sur le marché de leur signature esthétique. La forme de l'objet n'est plus indépendante de la forme intellectuelle ou éthique qui fonde les conditions de sa monstration. L'articulation du sens et de la forme d'apparition de l'œuvre, ou de l'expérience esthétique, s'installe au centre de la pratique de l'exposition et l'interroge en retour.

Les modifications apportées au dispositif artistique par les artistes de cette époque font que ceux-ci entrent en communication avec leurs spectateurs selon de nouvelles modalités. Les "objets dards" se présentent comme des provocations ou des propositions. Ils perdent l'aura qui nourrissait la fascination qu'exerçaient les peintures appartenant au régime traditionnel de la représentation, sorte de monde enclos dans les limites du cadre, paquet de sens s'imposant comme un en-soi intangible. Avec les " objets dard » et le "paysage ", on entre dans un dispositif de communication nouveau qui accorde au spectateur un rôle plus large qu'il n'avait jamais été. Certes le tableau traditionnel appelait de la part du spectateur un certain type de réponse, et donc de participation à la construction du sens, mais les dispositifs contemporains démultiplient cette ouverture de l'œuvre et du sens de manière à la fois consciente et violente.

De fait, en lançant cette notion de "paysage ", Smithson attaque directement l'illusion qui se cache au cœur du tableau comme media, cette surface orthogonale destinée à recevoir l'inscription du sens. Sans doute, dans son histoire, le " paysage » n'est-il pas étranger à la notion de surface, comme en atteste le fait que le terme a servi aussi bien pour décrire un tableau de paysage qu'un espace de nature s'offrant au regard. Mais en dehors de cette connivence avec la représentation et le tableau, l'objet " paysage »,tel que l'emploie Smithson, ouvre essentiellement sur un infini de variations qui sont déterminées par les modifications qu'y apportent le temps, la lumière et les principes physiques de l'entropie.

Cela est particulièrement vrai de la dimension géologique du "paysage ». En ce concentrant sur cet aspect, Smithson s'aventure au-delà de la surface pour creuser dans l'épaisseur de son objet, comme fait la balafre de Fontana. Toute surface cache et en même temps révèle un arrière plan. Si on prolonge cette évidence, encore en réaction chez l'artiste italien contre le privilège de la surface peinte de la Renaissance, on découvre un espace stratifié en profondeur dont les couches successives révèlent 


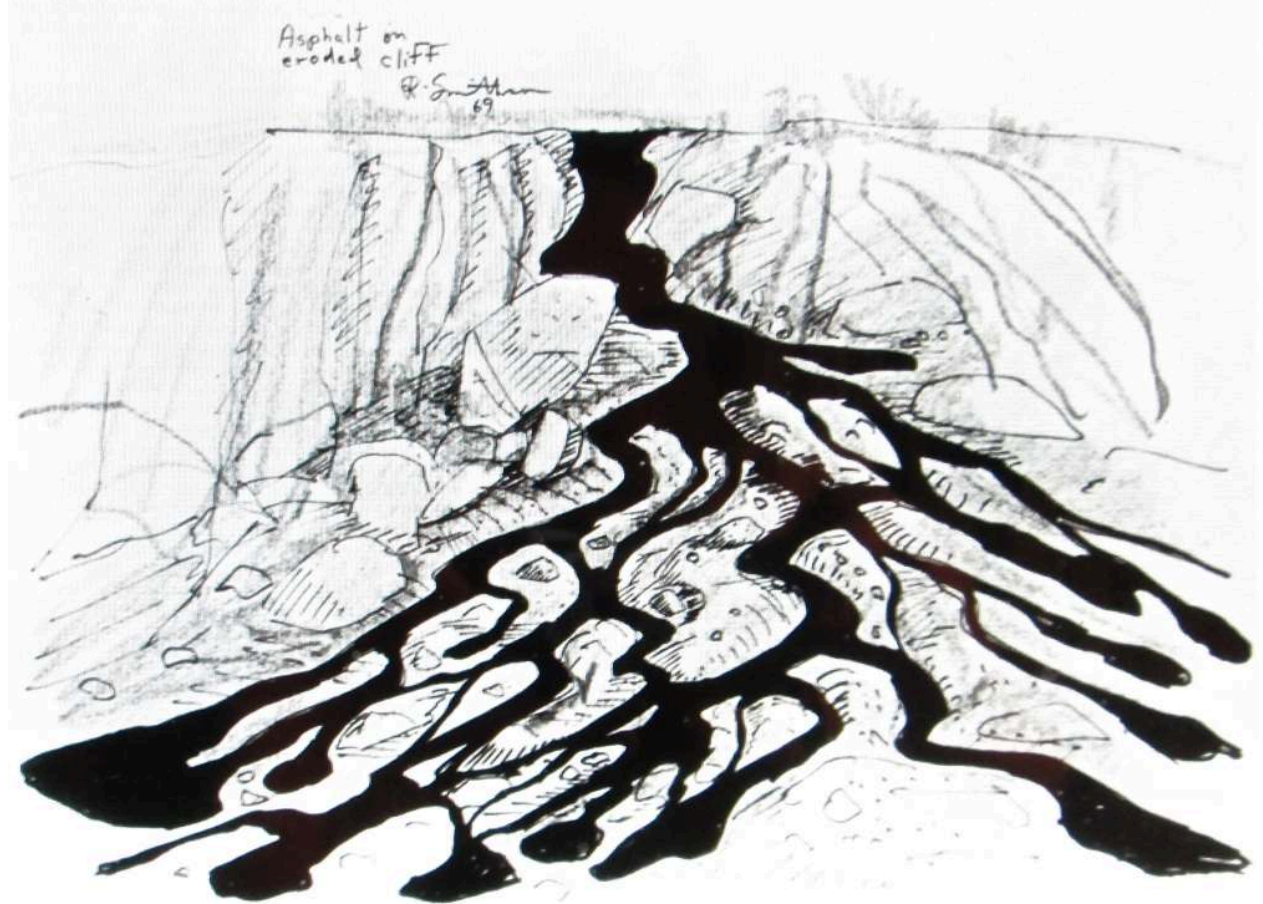

Fig. 1. Robert Smithson, Asfalt on eroded cliff, 1969. sans doute l'un des plus complexes, met en jeu et dépasse la dichotomie traditionnelle entre nature et culture. Le « paysage » est naturel par les matériaux qui le constituent : végétal, animal et minéral. Il obéit aux lois de la croissance végétative et à celles de la chimie du vivant (photosynthèse) en même temps qu'il subit les processus cosmiques d'érosion qui relèvent du principe d'entropie. Il se trouve donc à la charnière de deux phénomènes naturels contradictoires, opposant ses formes vitales d'organisation aux forces entropiques de désorganisation. Par ailleurs, le "paysage » est culturel par le cadrage perceptif qui le constitue et les modèles à travers lesquels cette perception se construit. Le " paysage » n'existe en effet que par rapport à un sujet spectateur, qui fait lui-même partie de la nature et se trouve, en tant que regardeur, construit dans et par l'évolution sociale et historique du regard. Cette articulation complexe de l'espace et du temps dans l'expérience sensible du « paysage »confère donc une place particulière au spectateur, à l'articulation des domaines de la nature et de la culture, dont ce faisant il relativise l'opposition conceptuelle.

Mais en faisant de l'expérience esthétique du spectateur l'origine même du « paysage », Smithson met en évidence une difficulté essentielle. Pour échapper au mutisme propre à toute expérience esthétique, le spectateur doit avoir recours à une forme de narration. C'est le récit, en effet, qui seul est capable de prendre en charge, dans l'écriture, l'expérience du temps. C'est lui qui permet, par la médiation de sa temporalité narrative, la figuration du développement de l'expérience. (Fig. 2) 


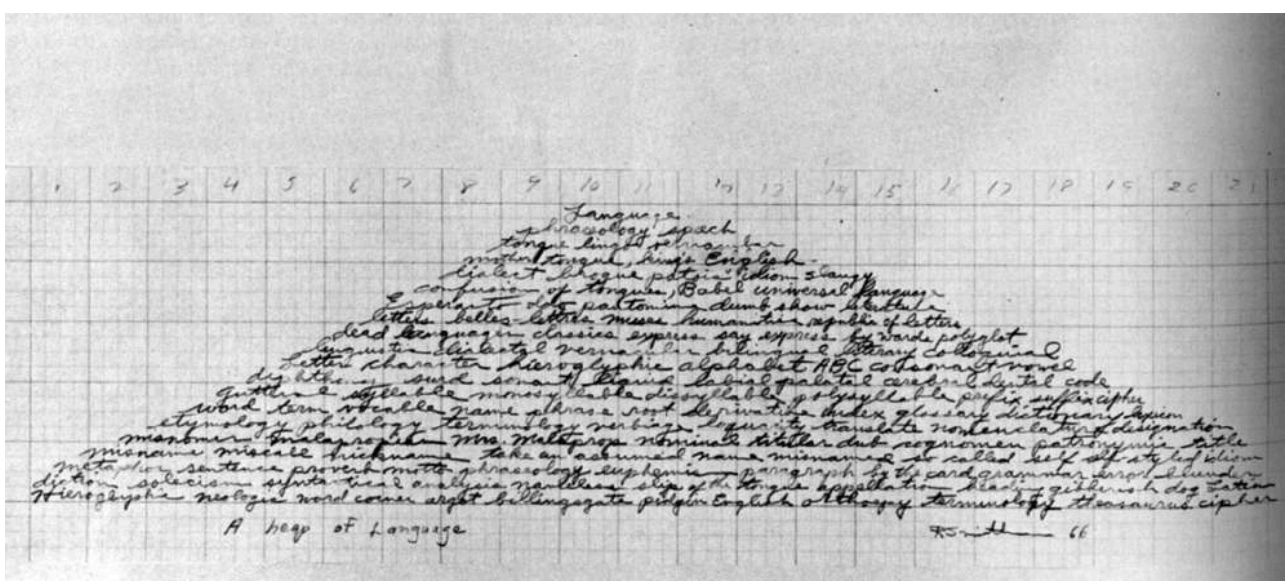

Fig. 2. Robert Smithson, A Heap of language, 1966. l'exception du bitume sombre qui se déroulait dans un paysage de plaine bordé au loin par des collines mais ponctué de cheminées, de fumées et de lumières colorées. ... La route et une grande partie du paysage étaient artificiels, cependant on ne pouvait pas parler d'œuvre d'art, pourtant cela produisit sur moi un effet que l'art n'avait jamais produit. Je n'ai d'abord pas compris pourquoi mais le résultat fut de m'affranchir de beaucoup d'idées. Il semblait qu'il y eut là une réalité qui n'avait jamais pu s'exprimer dans l'art. Cette expérience sur la route était quadrillée mais pas reconnue socialement. Je me disais qu'il était évident que c'était la fin de l'art, beaucoup de peintures ont l'air vraiment très picturales après cela. C'est une expérience impossible à cerner, il faut la vivre. J'ai découvert plus tard en Europe des pistes d'atterrissage désaffectées, œuvres à l'abandon, paysages surréalistes, sans fonctions, mondes fabriqués et sans tradition. Le paysage construit sans précédant culturel devient l'une de mes préoccupations. ${ }^{2}$

Ce récit produit des images qui se situent aux marges du discours artistique dans la mesure où les codes du monde des arts plastiques ne sont pas outillés pour restituer une telle expérience. Or ce que donne à sentir Tony Smith ce n'est rien moins que l'expérience du temps du monde à l'articulation de celle du temps du spectateur et dans les marges fragiles du langage. Smithson la définit comme «entropie ». Tony Smith, donne une forme langagière, elle-même à la limite de la possibilité de formuler, à quelque chose comme le sous-sol abyssal de l'expérience même du "paysage ». Les mots qu'il met en œuvre se trouvent affecté, dans leur existence comme langage, par une force entropique de désorganisation. Il leur arrive ce que subissent les strates physiques de la géologie : ils partent en lambeaux. Toutefois le récit reste malgré tout une manière de sauvegarder ce qui se disperse et s'effondre de la même façon que l'activité artistique rassemble en un «non-site » des pierres qui jadis faisaient corps 
avec la montagne. Les fragments épars que l'artiste réunit en un «non-site " prennent acte du morcellement tout en offrant une forme à son dépassement. Le récit procède de manière analogue avec les fragments d'un langage à la dérive.

L'esprit humain et la Terre sont constamment en voie d'érosion; des rivières mentales emportent des berges abstraites, les ondes du cerveau ébranlent des falaises de pensée, les idées se délitent en blocs d'ignorance et les cristallisations conceptuelles éclatent en dépôts de raison graveleuse. ${ }^{3}$

Smithson s'engage ici dans une stratégie qui vise à dépasser par une activité textuelle l'universelle désagrégation qui frappe du côté du langage comme de celui des choses. La pratique artistique constitue une réponse. Si quelque chose est possible, ce sera au prix de l'élaboration d'une poétique du « paysage » dont le principe résidera nécessairement dans la mise en forme du temps entropique.

Coordonner tout ce désordre et cette corrosion en motifs, grilles et subdivisions constitue un processus esthétique qui a jusqu'à présent été à peine esquissé. ${ }^{4}$

(Fig. 3, Fig. 4)
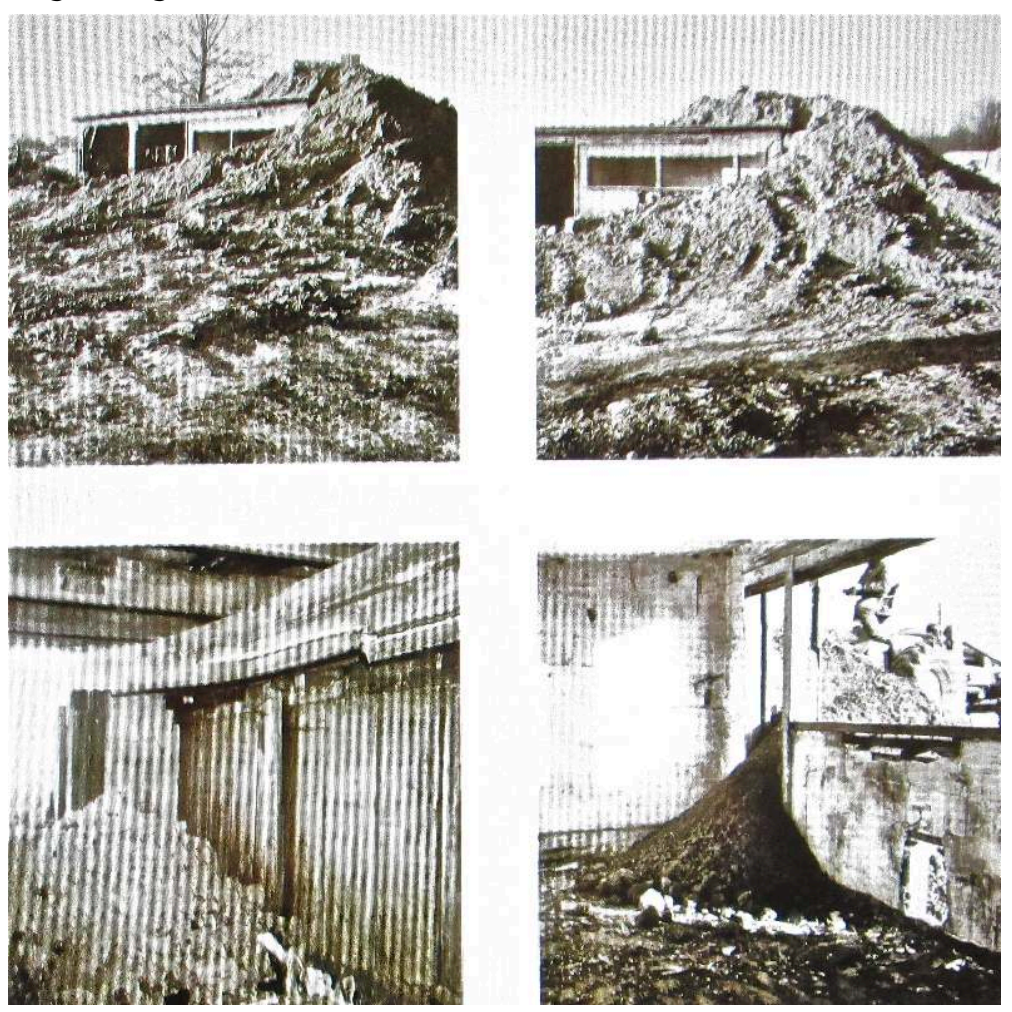

Fig. 3. Robert Smithson, Photograph of a partially buried woodshed, 1970. 


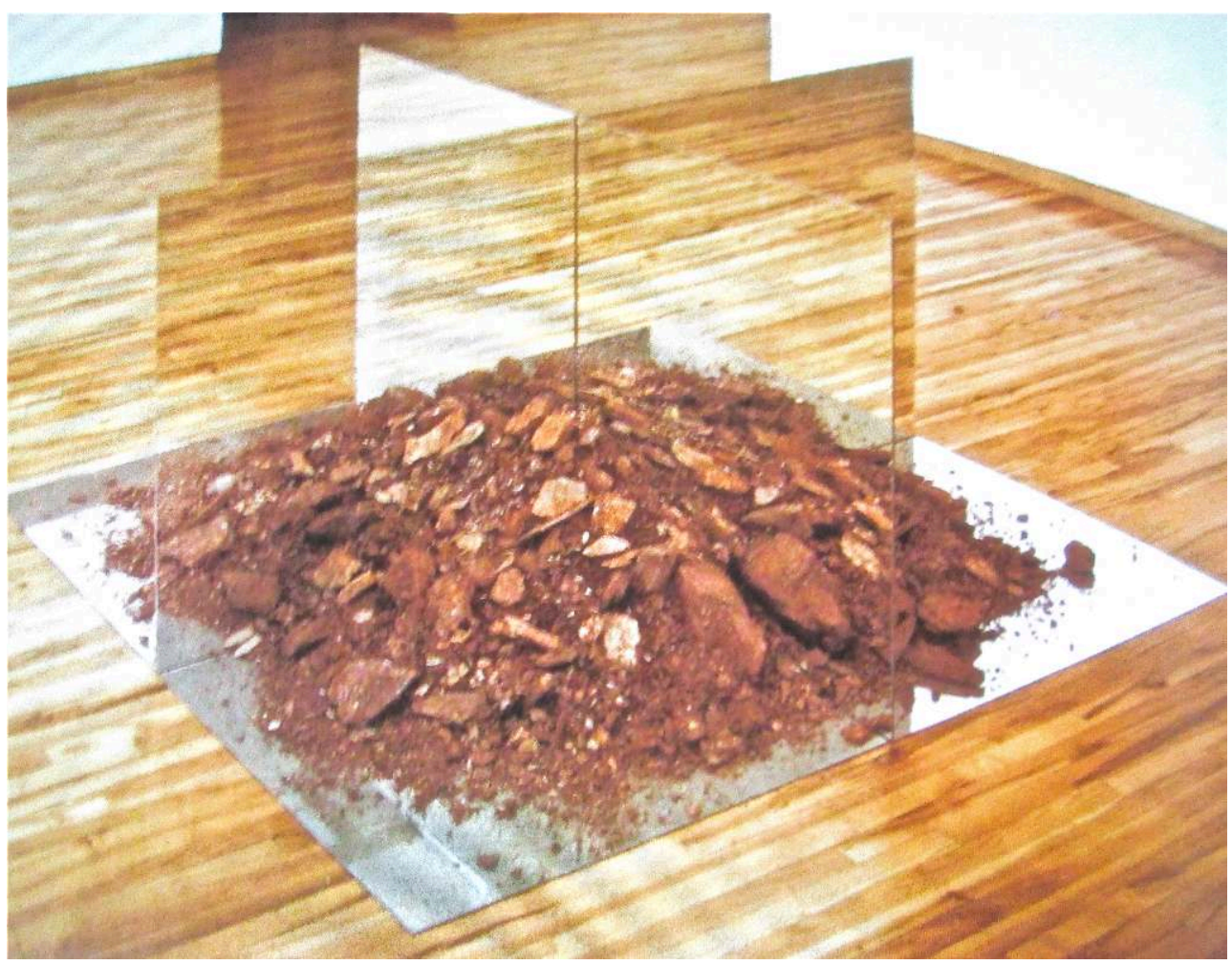

Fig. 4. Robert Smithson, Nonsite (Essen Soil and Mirrors), 1969.

Smithson sait qu'une telle poétique a déjà existé. Elle fut celle des ruines romantiques qui accompagnèrent, sur le mode nostalgique, l'expérience d'un temps destructeur. Le spectateur nostalgique s'identifiait alors lui-même à cette nature mortelle. Toutefois il ne pense pas que ce mode nostalgique soit la seule manière de capter et comprendre l'essence du temps entropique. Une autre poétique existe, qui consiste au contraire à sortir de l'attitude nostalgique et à instiller de la nég-entropie dans le système. En produisant des textes et des images, l'artiste invente des dispositifs et des artefacts qui, s'inscrivant dans le processus global de dégradation entropique, y réinscriront de la détermination et de la forme. Faire œuvre, répète Smithson, c'est ressaisir ce qui se défait dans une organisation inédite en prenant appui sur une énergie nouvelle, ou encore, c'est montrer que des processus organisationnels peuvent, dans le cadre limité du vivant, retarder les effets délétères du $2^{e}$. principe de la thermodynamique. Si Schrödinger a pu inventer la notion de nég-entropie, c'est parce qu'il était essentiel à ses yeux de distinguer du cadre général des lois de la thermodynamique, les processus vitaux. Ceux-ci, au sein même de la dégradation générale des formes organisées, produisent de nouvelles formes organisationnelles. Schrödinger les appelle principe vital.

Smithson, qui possédait une véritable curiosité scientifique et bénéficiait de très nombreuses lectures dans ce domaine, prend le monde physique comme un espace feuilleté d'expériences. Toutefois, le sous-sol auquel il porte une si constante attention pour sa soumission au principe de l'entropie n'est que l'horizon de la vie et de l'action humaines. Le "paysage", c'est chacun de nous ressaisi au sein du grand système entropique. La dialectique entre le monde physique et la vie, cette «dialectique de la nature » comme dit Smithson, construit un espace spécifique du poétique, absolument propre au vivant. L'expérience poétique du "paysage " passe donc par toute une 
gamme de stratégies nég-entropiques: un récit, une collecte de débris, une saisie photographique et ce faisant, elle construit un tiers espace et un tiers temps. Ce qu'une telle poétique fait advenir à l'existence relève du partage avec d'autres vivants de l'expérience entropique commune.

La singularité de ce tiers temps et de ce tiers espace, qui émergent pour Smithson à l'occasion de l'expérience du "paysage », rappelle les modalités que l'on trouve à l'œuvre dans le récit historique, lequel, dans son paradoxe propre, construit pour ses lecteurs une véritable histoire. Le récit paysager, qui comporte une narration proprement événementielle mais également toute une série de composants mémoriels, tels que traces, documents, images, établit une relation construite entre le temps individuel vécu et le temps cosmique.

Le « paysage » est donc un récit qui fait advenir le temps humain dans l'immensité du temps du monde. Autrement dit: un paysage, c'est du temps humain articulé à du temps cosmique, humain parce que construit dans un récit, dépendant de traces et de témoignages, cosmique parce que soumis au Principe de Carnot comme tout ce qui constitue l'univers. Tel que nous le montre Smithson, on pourrait dire enfin que le «paysage »est une expérience qui inscrit la liberté humaine dans l'horizon de la mort entropique.

On a jusqu'ici pensé le paysage selon deux axes de références : formelles, à partir des catégories de la géographie et esthétiques à partir de l'histoire de l'art, et singulièrement à travers la théorie de l'artialisation d'Alain Roger. ${ }^{5}$ (Fig. 5)

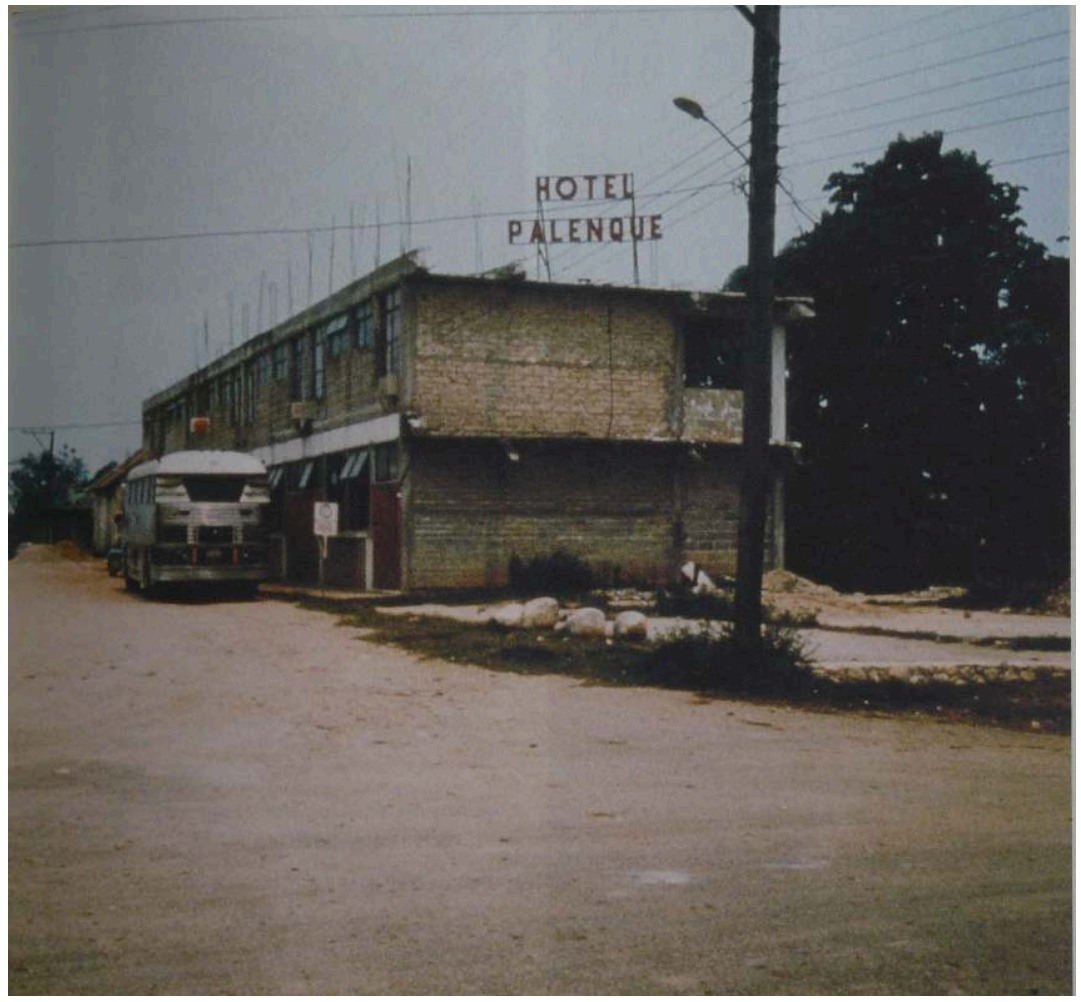

Fig. 5. Robert Smithson, Hôtel Palenque, 1969.

En positionnant le «paysage »au-delà de la représentation et de ses jeux de perspective illusionnistes, et en jetant le spectateur au cœur d'une expérience qui abandonne l'espace paramétré du musée, Smithson établit que ce qui constitue le «paysage » c'est d'être le lieu du partage de la temporalité entropique dans l'expérience. Faire 
l'expérience du "paysage", c'est se soumettre volontairement à la temporalité catastrophique qui entraîne le monde dans sa généralité et l'individu dans sa singularité. Mais s'il n'y avait que la participation contrainte de l'humain au cataclysme qui l'engloutit, le « paysage » ne serait pas devenu pour Smithson une sorte de solution au problème de l'art. C'est parce que,à partir des ruines, de la destruction et de l'obsolescence, le spectateur et l'artiste sont capables de construire un récit qu'apparaît une dialectique, un point d'Archimède à partir duquel l'entropie se retourne en négentropie, en pouvoir structurant de la survie. Une phrase tirée du récit de Smithson « Hôtel Palenque » affirme ce pouvoir de l'imagination et du dire : « on dirait que cette fenêtre improbable est la quintessence de la culture maya ${ }^{6}{ }^{6}$

Rapporté par l'artiste, mais aussi bien par tout spectateur, le « paysage » devient le lieu de cette tension, de cette dialectique où l'image et le texte sont des espaces différenciés d'inscription de la puissance structurante de la nég-entropie, au cœur même de l'effondrement général et du spectacle des ruines.

\section{NOTES}

1. Brian O'Doherty, White cube, L'espace de la galerie et son idéologie, Paris, Les Presses du réel, 2008.

2. Cf. Samuel Wagstaff Jr. « Talking with Tony Smith », in Artforum, décembre 1966.

3. «Une sédimentation de l'esprit : Earth projects », in Robert Smithson une rétrospective : le paysage entropique, Marseille - RMN, 1994, p. 192.

4. Idem.

5. Alain Roger, Court traité du paysage, Paris, Gallimard 1997.

6. Robert Smithson, Hôtel Palenque, (1969-1972).

\section{RÉSUMÉS}

Avec en toile de fond historique, Hiroshima et le devenir commercial de l'art, Robert Smithson va trouver dans l'idée d'entropie et de désordre croissant, qui excluent l'optimisme des pensées utopiques, une manière nouvelle de pratiquer l'art. Il est urgent pour lui de donner une nouvelle acuité au regard, celui de l'artiste comme celui des spectateurs. Avec une tendresse et une intelligence aiguës, Smithson invente une autre manière de regarder le paysage entropique, cette réalité symbolique de toutes les érosions, physiques comme intellectuelles. Dès lors, dessinant, photographiant et accumulant des traces, l'art réaffirme sa présence au cœur du désastre. 
INDEX

Mots-clés : Land Art, Paysage entropique, Robert Smithson

\section{AUTEUR}

\section{JACQUES LEENHARDT}

CRAL, Ecole des Hautes Etudes en Sciences Sociales, Paris.

Jacques Leenhardt est Directeur d'Études à l'École des Hautes Études en Sciences Sociales (Paris). Il travaille sur l'art et la littérature. Critique d'art et organisateur d'expositions, il a publié entre autres : Les Amériques latines en France (1992), Dans les Jardins de Roberto Burle Marx (1994-2011), Villette-Amazone (1996), Michel Corajoud, paysagiste (2000), Conscience du paysage. Le passant de Montreuil (2002), Jardins verticaux dans le monde entier, (2007), Le Fond et la Surface, photographies de Fabienne Barre (2008), Wifredo Lam, Essai monographique, 2009, La poétique du bord, photographies de Olivier Amsellem (2010), 